\title{
Fatores epidemiológicos do dengue na região central do estado do Rio Grande do Sul, Brasil, 2007 - 2010
}

\author{
Epidemiological features of dengue in the central region of Rio Grande do Sul state, Brazil, \\ from 2007 to 2010
}

\author{
Ivan Xavier Gabriela Valle ${ }^{\mathrm{II}}$ Danieli Lunkes ${ }^{\mathrm{II}}$ Anderson Spohr Nedel ${ }^{\mathrm{III}}$ Vagner Anabor ${ }^{\mathrm{III}}$ \\ Marli Matiko Anraku de Campos ${ }^{\text {II }}$ Sônia de Avila Botton ${ }^{\text {IV }}$ Miriam Cabrera Corvelo Delboni ${ }^{\text {II }}$ \\ Luís Antonio Sangioni ${ }^{I^{*}}$
}

\begin{abstract}
O objetivo deste estudo foi avaliar fatores epidemiológicos do dengue, nos municípios da $4^{a}$ Coordenadoria Regional de Saúde/RS (4⿳亠口冋 $C R S / R S)$, nos anos de 2007 a 2010. Trata-se de um estudo descritivo, empregando como variáveis: município de notificação; ano de notificação; sexo e faixa etária do paciente; índice de infestação predial (IIP) e índice de Breteau (IB). Foram constatados 13 casos importados da doença, em cinco municípios, sendo a maior ocorrência em Santa Maria. Houve notificação de casos em ambos os sexos e em diferentes faixas etárias. Focos positivos do Aedes aegypti foram encontrados em armadilhas instaladas em pontos estratégicos de Santa Maria e Santiago. O IIP e IB, em ambos os municípios, não representaram risco à saúde pública. Conclui-se, portanto, que as ações de vigilância em saúde devem ser fomentadas com o objetivo de orientar as práticas de controle do vetor, bem como promover a educação sanitária e ambiental, a fim de impedir a instalação de casos autóctones nos municípios estudados.
\end{abstract}

Palavras-chave: Ae. aegypti, vigilância em saúde, saúde pública, controle.

\section{ABSTRACT}

The aim of this study was to evaluate the epidemiological issues of dengue in the counties of the $4^{\text {th }}$ Coordenadoria Regional de Saúde (government agency to surveillance on public health) of Rio Grande do Sul state, Brazil, during 2007 to 2010. This is a descriptive study, using as variables: cities where dengue was reported; year; sex and age of the patient; infestation (IIP) and Breteau (BI) index. A total of 13 imported cases of the dengue was reported in five cities and higher occurrence was in Santa Maria city. There were notifications of dengue in both sex and in different age groups. Positive foci of Aedes aegypti were found in the traps at strategic points in both cities, Santa Maria and Santiago. BII and BI varied during the studied period in both cities, and, so far, they did not constitute a risk to public health. We conclude that the health surveillance should be enhanced with the purpose of guiding vector control practices, as well as promote health and environmental education in order to prevent the installation of autochthonous dengue cases in the cities studied.

Key words: Ae. aegypti, health surveillance, public health, control.

Dengue é causado por um Flavivirus, da família Flaviviridae, que apresenta quatro sorotipos antigenicamente distintos entre si: DEN-1, DEN-2, DEN3 e DEN-4. Aedes aegypti é considerado o principal transmissor dessa arbovirose no Brasil (TAUIL, 2002). Além desse mosquito, Ae. albopictus também é um vetor do vírus em áreas rurais e urbanas em vários países, incluindo o Brasil. Contudo, ainda não está associado à transmissão do vírus a hospedeiros humanos susceptíveis (CHIARAVALLOTI NETO et al., 2002; PASSOS et al., 2003).

\footnotetext{
'Secretaria da Saúde de Santa Maria, Superintendência de Vigilância em Saúde, Vigilância Ambiental, Santa Maria, RS, Brasil. IIPrograma de Educação pelo Trabalho em Vigilância Sanitária (PET/VISA), Departamento de Medicina Veterinária Preventiva (DMVP), Centro de Ciências Rurais (CCR), Universidade Federal de Santa Maria (UFSM), Av. Roraima, 1000, Prédio 44 (CCRII), sala 5149, Campus Universitário, Camobi, 97105-900, Santa Maria, RS, Brasil. E-mail: lasangioni@gmail.com. *Autor para correspondência.

IIIDepartamento de Física, Centro de Ciências Naturais e Exatas, UFSM, Santa Maria, RS, Brasil.

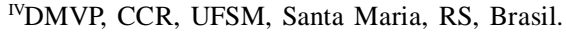


Em 2007, foram registrados os primeiros casos humanos autóctones do dengue no RS. Em 2008 e 2009, apenas casos importados foram notificados e, em 2010, uma epidemia da doença ocorreu na região das Missões, no noroeste do estado (BARONI \& OLIVEIRA, 2009). Essa região, limítrofe ao estado de Santa Catarina e à Argentina, é contígua à região central do RS, sendo considerada uma importante região endêmica da doença no estado. Santa Maria é um importante município da região central reunindo grande número de instituições de ensino superior, unidades militares e referência em serviços de saúde. Dessa forma, o município possui alta concentração de pessoas e veículos em circulação e também vem apresentando, nos últimos anos, aumento contínuo de moradias e estabelecimentos comerciais (BRASIL, 2010). Consequentemente, esses fatores favorecem o risco de importação do Ae. aegypti e à circulação do vírus do dengue. O objetivo deste trabalho foi analisar os dados epidemiológicos referentes ao dengue em municípios da região central do RS, no período de 2007 a 2010.

O estudo foi realizado na 4 a $\mathrm{CRS} / \mathrm{RS}$, com sede em Santa Maria, e abrangeu 31 municípios da região central do RS. Trata-se de um estudo descritivo, tendo sido analisadas as variáveis: município de notificação; ano de notificação; sexo e faixa etária do paciente; índice de infestação predial (IIP); e índice de Breteau (IB). Os dados analisados procederam das seguintes fontes: relatórios da Secretaria Municipal de Saúde do Município de Santa Maria; Sistema de Informação de Febre Amarela e Dengue (SISFAD); e Sistema Nacional de Agravos de Notificação (SINAN).

No período de 2007 a 2010, foram notificados 13 casos de dengue em cinco $(16,1 \%)$ dos 31 municípios da 4 CRS/RS (Júlio de Castilhos, Nova Palma, Santa Maria, Santiago e Tupanciretã). A investigação dos casos sugeriu serem todos importados e pertencentes ao sorotipo DEN-1, sendo os pacientes infectados principalmente nos estados de RO, AC, AP, MG, MS, $\mathrm{GO}$ e no interior do noroeste do RS, onde havia registros de casos autóctones da doença, segundo informação da 4a CRS/RS (dados não mostrados). Em 2007, ocorreram cinco casos em quatro municípios da regional; em 2008, foram notificados dois casos em Santa Maria; em 2009, não houve registro da doença; e, no ano de 2010, foram diagnosticados cinco casos em Santa Maria e um em Santiago. Os casos de dengue foram distribuídos em todas as faixas etárias estudadas, abrangendo de um a 69 anos de idade. No entanto, em Santa Maria, não houve registro na faixa de 30 a 39 anos. Segundo GONÇALVES NETO \& REBELO (2004), todas as idades são susceptíveis ao vírus do dengue, porém o padrão mais observado é a ocorrência de casos nas faixas etárias mais elevadas. Neste estudo, verificou-se que a maioria dos indivíduos (85\%) acometidos eram adultos (11/13), com maior mobilidade urbana. $\mathrm{Na} 4^{\mathrm{a}} \mathrm{CRS} / \mathrm{RS}$, foram registrados casos em pacientes de ambos os sexos. RIBEIRO et al. (2006) demonstraram maior predominância do dengue em mulheres nas faixas etárias entre 20 a 39 anos. VASCONCELOS et al. (1993) sugerem que a maior permanência da mulher no intradomicílio e/ou peridomicílio favorece o contato com os criadouros do vetor. No entanto, neste estudo, não se estabeleceu a relação entre a ocorrência de casos em ambos os sexos.

Larvas do Ae. aegypti foram encontradas em bairros e na região central de Santa Maria, durante os anos de 2007 e 2010. Na cidade de Santiago, foram detectados focos positivos do Ae. aegypti e Ae. albopictus em residências e depósitos de água no período de 2007 a 2010. Também foram detectados focos positivos de larvas de Ae. albopictus em armadilhas instaladas em pontos estratégicos (borracharias, rodoviária, cemitérios) em 15 municípios da 4- CRS/RS. Os criadouros artificiais, especialmente aqueles encontrados nos vasos de plantas, pneus abandonados a céu aberto, entre outros, todos com coleções de água, são importantes fatores na manutenção de altos níveis de infestação dos vetores Ae. aegypti e Ae. albopictus. Além disso, o espaço social da forma que está organizado permite a interação entre o vetor, vírus e ser humano (PASSOS et al., 2003; MONDINO \& CHIARAVALLOTI NETO, 2007). Ressalta-se que $\boldsymbol{A} \boldsymbol{e}$. albopictus vem ocupando quase todas as áreas urbanizadas, demonstrando alto potencial de dispersão vetorial, uma vez que sua população concentrava-se em ambientes silvestres (CHIARAVALLOTI NETO et al., 2002). Neste estudo, foi detectada a presença de ambos os mosquitos, sendo que a população de larvas de Ae. albopictus foi predominante em relação ao $\boldsymbol{A} \boldsymbol{e}$. aegypti. Em Santa Maria, encontrou-se Ae. albopictus na zona urbana, provavelmente devido às condições geográficas da cidade, que se encontra em área de depressão circundada por ambiente silvestre, possibilitando a multiplicação do vetor. PASSOS et al. (2003) relataram haver competição intra-específica entre ambos os mosquitos presentes em um mesmo ecótopo. Isto, possivelmente, pode contribuir para a diminuição da transmissão do vírus do dengue para a população da região central do RS, uma vez que, nas Américas, ainda não foi atribuída uma eficiente transmissão do agente etiológico pelo Ae. albopictus.

No período estudado, avaliaram-se $32.401 \mathrm{e}$ 135.650 imóveis em Santa Maria e Santiago, respectivamente. Com relação ao índice de infestação predial (IIP) e índice de Breteau (IB), em Santa Maria, 
não houve registro de ambos os índices para o $\boldsymbol{A e}$. aegypti, enquanto que os índices IIP e IB registrados para o Ae. albopictus foram, respectivamente, 1,03 e 1,03 (2007) e 0,47 e 0,53 (2010). No município de Santiago, para o Ae. aegypti, foram encontrados os mesmos valores para ambos os índices, sendo: 0,04 (2007); 0,03 (2008); 0,02 (2009)e, 0,07 (2010). No entanto, para Ae. albopictus, índices IIP e IB iguais a 0,05 foram registrados somente no ano de 2007.

GOMES (1998) relatou que, para caracterizar situação de iminente perigo à saúde pública, em se tratando do dengue, devem ser considerados a presença do vetor e IIP e IB maiores que 1 e 5, respectivamente. Pelos índices obtidos neste estudo, para ambos os mosquitos, a situação identificada nos municípios de Santa Maria e Santiago não representa risco para a saúde pública, o que, em parte, pode explicar a ocorrência de casos importados e não autóctones do período analisado.

Durante os anos de 2007 a 2010, houve readequação administrativa no quadro dos agentes de saúde de Santa Maria e Santiago (4- CRS/RS, comunicação verbal), o que pode ter influenciado nas limitações das atividades de controle de vetores, incluindo desde a falta de recursos humanos para o desenvolvimento das ações até a qualidade do trabalho executado. Segundo BAGLINI et al. (2005), é importante que as medidas de controle do vetor sejam executadas com a participação efetiva dos profissionais ligados aos órgãos de gestão de controle de endemias, auxiliados pela população local. Este fato contribui para diminuir os níveis da infestação, uma vez que há tendência da população em não aderir às medidas profiláticas quando não há a efetiva ação dos agentes em promover a educação sanitária continuada.

Nos municípios, são realizadas mensalmente as seguintes atividades: levantamento de índice amostral; visitas domiciliares; pesquisa entomológica nos pontos estratégicos com ovitrampas ou larvitrampas; atividades de conscientização e participação comunitária na promoção do saneamento domiciliar e inspeção da coleta pública de lixo. Nas armadilhas localizadas nos depósitos e imóveis, onde foram identificadas larvas do $\boldsymbol{A e}$. aegypti, foi realizada delimitação de foco e tratamento com organofosforado em $100 \%$ dos imóveis incluídos em um raio de até 300 metros, conforme preconizado pelas normas do Plano Nacional de Controle do Dengue (BRASIL, 2001).

Nos municípios da 4르 CRS/RS, foram registrados apenas casos importados da doença. Ressalta-se a necessidade de maior atenção da vigilância epidemiológica e entomológica para que as infestações vetoriais e/ou os casos da doença sejam precocemente identificados. Conclui-se, portanto, que as ações de vigilância em saúde devem ser fomentadas com o objetivo de orientar as práticas de controle do vetor, bem como promover a educação sanitária e ambiental, a fim de impedir a instalação de casos autóctones nos municípios estudados.

\section{REFERÊNCIAS}

BAGLINI, V. et al. Atividades de controle do dengue na visão de seus agentes e da população atendida, São José do Rio Preto, São Paulo, Brasil. Cadernos de Saúde Pública, v.21, n.4, p.1142-1152, 2005. Disponível em: <http://www.scielo.br/pdf/ csp/v21n4/16.pdf>. Acesso em: 18 jul. 2012.

BARONI, C.J.; OLIVEIRA, T.B. Aspectos epidemiológicos da febre clássica da dengue, em Giruá - RS. Revista Brasileira de Análises Clínicas, v.41, n.4, p.289-293, 2009. Disponível em: <http://www.sbac.org.br/pt/pdfs/rbac/rbac_41_04/ rbac_41_4_010.pdf>. Acesso em: 18 jul. 2012.

BRASIL. Dengue instruções para pessoal de combate ao vetor: manual de normas técnicas. 3.ed. Brasília-DF. Ministério da Saúde, Fundação Nacional de Saúde, 2001. 84p. (Guia Técnico). Disponível em: <http://bvsms.saude.gov.br/bvs/ publicacoes/funasa/man_dengue.pdf $>$. Acesso em: 18 jul. 2012.

BRASIL. Censo demográfico. Brasília-DF. Ministério do Planejamento e Gestão, Instituto Brasileiro de Geografia e Estatística (IBGE), 2010. Disponível em: <http://www.ibge.gov.br/ home/estatistica/populacao/censo2010/sinopse/ default_sinopse.shtm>. Acesso em: 25 jul. 2011.

CHIARAVALlOTI NETO, F. et al. Aedes albopictus na região de São José do Rio Preto, SP: estudo da sua infestação em área já ocupada pelo Aedes aegypti e discussão de seu papel como possível vetor de dengue e febre amarela. Revista da Sociedade Brasilera de Medicina Tropical v.35, n.4, p.351-357 2002. Disponível em: <http://www.scielo.br/scielo.php?script=sci_arttext\&pid=S003786822002000400012\&lng=pt\&nrm=iso>. Acesso em: 27 jul. 2011. doi: $10.1590 / \mathrm{S} 0037-86822002000400012$.

CHIARAVALlOTi NETO, F. et al. Controle do vetor do dengue e participação da comunidade de Catanduva, São Paulo, Brasil. Cadernos de Saúde Pública, v.19, p.1739-1749, 2003. Disponível em: <http://www.scielo.br/pdf/csp/v19n6/ a18v19n6.pdf>. Acesso em: 18 jul. 2012.

DEPRADINE C.A.; LOVELL E.H. Climatological variables and the incidence of dengue fever in Barbados. Internatinal Journal of Enviromental research and Public Health, v.14, p.429-441, 2004. Disponível em: <http://www.ncbi.nlm.nih.gov/ pubmed/15545038>. Acesso em: 10 mar. 2012. doi: 10.1080/ 09603120400012868 .

GOMES, A.C. Medidas dos níveis de infestação urbana para Aedes (Stegomyia) aegypti e Aedes (Stegomyia) albopictus em programa de vigilância entomológica. Informe Epidemiológico do SUS, v.7, p.49-57, 1998. Disponível em: <http://portal.saude.gov.br/portal/ arquivos/pdf/iesus_vol7_3_medidas.pdf>. Acesso em: 18 jul. 2012.

GONÇALVES NETO, V.S.; REBÊLO, J.M.M. Aspectos epidemiológicos do dengue no município de São Luis, Maranhão, Brasil, 1997-2002. Cadernos de Saúde Pública, v.20, 
p.1424-1431, 2004. Disponível em: <http://www.scielo.br/pdf/ csp/v20n5/39.pdf>. Acesso em: 18 jul. 2012.

PASSOS, R.A. et al. Dominância de Aedes aegypti sobre Aedes albopictus no litoral sudeste do Brasil. Revista de Saúde Pública, v.37, p.729-734, 2003. Disponível em: <http:// www.scielosp.org/pdf/rsp/v37n6/18015.pdf >. Acesso em: 18 jul. 2012.

REITER P.W. Vector biology and arboviral recrudescense. In: MONATH, T.P. (Ed.). The arboviruses: epidemiology and ecology. Boca Raton (FL): CRC, 1988. p.245-255.

RIBEIRO, A.F. et al. Associação entre incidência de dengue e variáveis climáticas. Revista de Saúde Pública, v.40, n.4, p.671-676, 2006.
Disponível em: <http://portal.saude.gov.br/portal/arquivos/kitdengue/ epidemiologia/textos/associacaodengueevariaveisclimaticas.pdf $>$. Acesso em: 18 jul. 2012.

TAUIL, P.L. Aspectos críticos do controle do dengue no Brasil. Caderno de Saúde Pública, v.18, n.3, p.867-871, 2002. Disponível em: <http://www.scielo.br/pdf/csp/v18n3/9314.pdf>. Acesso em: 17 fev. 2011

VASCONCELOS, P.F.C. Epidemia de febre clássica de dengue causada pelo sorotipo 2 em Araguaína, Tocantins, Brasil. Revista do Instituto de Medicina Tropical de São Paulo, v.35, p.141-148, 1993. Disponível em: <http://www.scielo.br/ pdf/rimtsp/v35n2/a05v35n2.pdf>. Acesso em: 18 jul. 2012. doi: $10.1590 / \mathrm{S} 0036-46651993000200005$. 


\title{
ERRATA
}

Artigo publicado com a grafia do autor Vagner Anabor incorreta.

\section{Fatores epidemiológicos do dengue na região central do estado do Rio Grande do Sul,} Brasil, 2007 - 2010

\author{
Epidemiological features of dengue in the central region of Rio Grande do Sul state, \\ Brazil, from 2007 to 2010
}

\author{
Ivan Xavier $^{\mathrm{I}}$ Gabriela Valle $^{\mathrm{II}}$ Danieli Lunkes ${ }^{\mathrm{II}}$ Anderson Spohr Nedel ${ }^{\mathrm{III}}$ Vagner Anabor ${ }^{\mathrm{III}}$ \\ Marli Matiko Anraku de Campos ${ }^{\mathrm{II}}$ Sônia de Avila Botton ${ }^{\mathrm{IV}}$ Miriam Cabrera Corvelo Delboni ${ }^{\mathrm{II}}$ \\ Luís Antonio Sangioni ${ }^{\mathrm{II}^{*}}$
}

Para a versão correta, acesse: http://www.scielo.br/pdf/cr/v43n1/a0413cr6059.pdf

ISecretaria da Saúde de Santa Maria, Superintendência de Vigilância em Saúde, Vigilância Ambiental, Santa Maria, RS, Brasil. IIPrograma de Educação pelo Trabalho em Vigilância Sanitária (PET/VISA), Departamento de Medicina Veterinária Preventiva (DMVP), Centro de Ciências Rurais (CCR), Universidade Federal de Santa Maria (UFSM), Av. Roraima, 1000, Prédio 44 (CCRII), sala 5149, Campus Universitário, Camobi, 97105-900, Santa Maria, RS, Brasil. E-mail: lasangioni@gmail.com. *Autor para correspondência.

IIIDepartamento de Física, Centro de Ciências Naturais e Exatas, UFSM, Santa Maria, RS, Brasil.

${ }^{\mathrm{IV}}$ DMVP, CCR, UFSM, Santa Maria, RS, Brasil. 